\section{(C) OPEN ACCESS}

\title{
Randomised trial and open-label extension study of an anti-interleukin-6 antibody in Crohn's disease (ANDANTE I and II)
}

\author{
Silvio Danese, ${ }_{1}^{1}$ Séverine Vermeire, ${ }^{2}$ Paul Hellstern, ${ }^{3}$ Remo Panaccione, ${ }^{4}$ \\ Gerhard Rogler, ${ }_{1}^{5}$ Gerald Fraser, ${ }_{1}^{6}$ Anna Kohn, ${ }^{7}$ Pierre Desreumaux, ${ }^{8}$ Rupert W Leong, ${ }^{9}$ \\ Gail M Comer, ${ }_{10}^{10}$ Fabio Cataldi, ${ }^{10}$ Anindita Banerjee, ${ }^{11}$ Mary K Maguire, ${ }^{12}$ Cheryl Li, ${ }^{11}$ \\ Natalie Rath, ${ }^{12}$ Jean Beebe, ${ }^{11}$ Stefan Schreiber ${ }^{13}$
}

- Additional material is published online only. To view please visit the journal online (http://dx.doi.org/10.1136/ gutjnl-2017-314562).

For numbered affiliations see end of article.

\section{Correspondence to}

Dr Silvio Danese, Department of Gastroenterology, Humanitas Clinical and Research Center and Humanitas University, Via Manzoni 56, 20089 Rozzano, Milan, Italy; sdanese@hotmail. com

SD and SS contributed equally.

Received 25 May 2017 Revised 20 November 2017 Accepted 22 November 2017 Published Online First 15 December 2017
Check for updates

To cite: Danese $S$,

Vermeire $S$, Hellstern $P$, et al. Gut 2019;68:40-48.

\section{ABSTRACT}

Objective Neutralising pro-inflammatory interleukin-6 (IL-6) may effectively treat Crohn's disease (CD). Effects of PF-04236921, an anti-IL-6 antibody, in adults with CD are reported.

Design Parallel-group, dose-ranging, double-blind trial with 4-week screening and 12-week treatment periods. After induction, patients entered 28-week follow-up or 48-week open-label extension (OLE) with 28-week follow-up. Adults with confirmed CD and inadequate response to anti-tumour necrosis factor (TNF) therapy were included. Induction study: 249 patients randomised 1:1:1:1 to placebo, PF-04236921 10,50 or $200 \mathrm{mg}$ by subcutaneous injection on days 1 and 28. OLE study: PF$0423692150 \mathrm{mg}$ every 8 weeks up to six doses followed by 28-week follow-up.

Results 247 patients were randomised and received treatment in the induction study. The $200 \mathrm{mg}$ dose was discontinued due to safety findings in another study (NCT01405196) and was not included in the primary efficacy analysis. Crohn's Disease Activity Index (CDAI)70 response rates with PF-04236921 50 mg were significantly greater than placebo at weeks $8(49.3 \%$ vs $30.6 \%, \mathrm{P}<0.05)$ and $12(47.4 \%$ vs $28.6 \%, \mathrm{P}<0.05)$ and met the primary end point. Week 12 CDAl remission rates with PF-04236921 $50 \mathrm{mg}$ and placebo were $27.4 \%$ and $10.9 \%$, respectively (16.5\% difference; $\mathrm{P}<0.05)$. 191 subjects received treatment in the OLE. Common treatment-emergent and serious adverse events in both studies included worsening CD, abdominal pain and nasopharyngitis.

Conclusions PF-0423692150 mg induced clinical response and remission in refractory patients with moderate-to-severe CD following failure of anti-TNF therapy. GI abscess and perforation were observed, a specific focus of attention during future clinical development.

Trial registration number NCT01287897 and NCT01345318.

\section{INTRODUCTION}

Crohn's disease (CD) is a chronic inflammatory condition of the GI tract with no known cure. Antibody-based therapies directed against tumour necrosis factor (TNF) ${ }^{1-3}$ or interleukin 12/

\section{Significance of this study}

What is already known on this subject?

- Crohn's disease (CD) has no known cure.

- The cytokine interleukin-6 (IL-6) has multiple pro-inflammatory effects such as inhibition of apoptosis in mucosal T-cells and is a logical target for treating $C D$.

- PF-04236921 is a fully human immunoglobulin G2 monoclonal antibody that binds to human IL-6.

What are the new findings?

- CD Activity Index (CDAI)-70 response and remission rates with PF-04236921 $50 \mathrm{mg}$ were significantly greater than placebo in the induction study.

- Common treatment-emergent and serious adverse events in both studies included worsening $\mathrm{CD}$, abdominal pain and nasopharyngitis. The benefit/risk profile for PF-04236921 is acceptable for the continued development of this treatment in this refractory CD population. Signals of GI perforation and abscess require careful consideration during future clinical development.

How might it impact on clinical practice in the foreseeable future?

- These studies are relevant in light of the limited therapeutic options available to difficult-totreat patients with $C D$ who experience primary non-response, relapse or intolerance to antitumour necrosis factor therapy.

- In addition, the studies support growing understanding of the potential of targeting IL- 6 for the treatment of inflammatory conditions.

interleukin $23 \mathrm{p} 40$ subunit antibody ${ }^{4}$ or integrins $\left(\alpha_{4} \beta_{7} \alpha_{4} \beta_{1} \text { integrin, } \alpha_{4} \beta_{7} \text { integrin }\right)^{4}$ have demonstrated efficacy in moderate-to-severe CD. Despite these options, up to $50 \%$ of patients experience primary non-response and more than 50\% can lose response over time to anti-TNF maintenance therapy, so that dose adjustment, or switch to a different anti-TNF or non-anti-TNF therapy, is 
required. ${ }^{5}$ This leaves a significant unmet need in the treatment of non-responsive patients, particularly after therapy with anti-TNF antibodies.

The cytokine interleukin-6 (IL-6) has multiple pro-inflammatory effects such as inhibition of apoptosis in mucosal T-cells and is a logical target for treating $\mathrm{CD} .{ }^{6} \mathrm{~A}$ small $(\mathrm{n}=36) 12$-week, placebo-controlled pilot study of the IL-6 receptor inhibitor, tocilizumab, in moderate-to-severe CD suggested a clinical benefit. $^{7}$

PF-04236921 is a fully human immunoglobulin G2 monoclonal antibody that binds to human IL- 6 and has a half-life of 36-51 days. In phase I trials in healthy volunteers and patients with rheumatoid arthritis (protocol B0151001, NCT00838565 and NCT01166555), intravenous and subcutaneous (SC) PF-04236921 was well tolerated and caused sustained suppression of C-reactive protein (CRP), a marker for inflammation that is transcriptionally controlled by IL- $6 .{ }^{8}$ PF-04236921 has also been investigated in a phase II trial in patients with systemic lupus erythematosus (SLE; NCT01405196). ${ }^{9}$ While the study did not meet the primary end point, improvement was noted in the primary as well as key secondary end points with $10 \mathrm{mg}$.

In this phase II trial, the efficacy, pharmacokinetics, safety, tolerability and immunogenicity of PF-04236921 were assessed in patients with moderate-to-severe $\mathrm{CD}$, who were inadequate responders to anti-TNF therapy. It was followed by an openlabel extension (OLE) study with the primary objective of assessing the long-term safety, tolerability and immunogenicity of PF-04236921.

\section{METHODS}

\section{Patients}

In this multicentre, phase II, randomised study, patients were aged 18-75 years and had moderate-to-severe CD (Crohn's Disease Activity Index (CDAI) score 220-450) with CRP $\geq 5.0 \mathrm{mg} / \mathrm{L}$ and ulceration demonstrated by colonoscopy within 8 weeks before screening. All patients had failed $\geq 1$ anti-TNF therapy due to primary non-response, loss of response, intolerance or another reason. Permitted and prohibited treatments and study exclusion criteria are described in the online supplementary file.

Patients were enrolled in the OLE study if they had completed the blinded 12-week induction period. Patients were excluded from the OLE study if they had experienced a serious adverse event (SAE) related to treatment with PF-04236921 during the primary study or if there was a contraindication to ongoing treatment.

Protocols were approved by the relevant institutional review boards or ethics committees. Both studies complied with the ethical principles of the Declaration of Helsinki and the International Conference on Harmonization Good Clinical Practice Guidelines. Safety was reviewed throughout the studies by an external data monitoring committee (DMC). All patients provided written, informed consent.

\section{Study design}

This parallel-group, dose-ranging, double-blind phase II induction trial (ClinicalTrials.gov identifier: NCT01287897) involved a 4-week screening period and a 12-week treatment period. Patients were planned to be randomised 1:1:1:1 to receive placebo or PF-04236921 10, 50 or $200 \mathrm{mg}$ SC, on days 1 and 28. Randomisation was performed using a computer-generated randomisation scheme, with stratification by background immunosuppressive therapy (azathioprine, six mercaptopurine, methotrexate or none) and reason for prior anti-TNF failure.
Dose regimens were selected to provide a range of CRP suppression, based on pharmacokinetic pharmacodynamic modelling of phase I data.

After the induction period, patients entered either a 28-week follow-up period or the 48-week OLE study to receive PF-04236921 $50 \mathrm{mg}$ every 8 weeks followed by a 28 -week follow-up period (NCT01345318). To enter the OLE, patients were required to complete the induction period but were not required to be considered responders. In the OLE, a one-time dose escalation to $100 \mathrm{mg}$ was allowed for non-responders starting at week 8 . If the patient failed to respond within 8 weeks after dose escalation, they were discontinued from the active treatment period. Patients continued to be monitored every 4 weeks through to the final OLE study evaluation at week 76 .

All authors had access to the study data, and reviewed and approved the final manuscript.

\section{Efficacy assessments}

The primary end point for the induction study was CDAI-70 response rate (proportion of patients achieving a $\geq 70$-point reduction in CDAI score) at weeks 8 or 12. Analysis of the efficacy end points was prespecified based on the modified intentto-treat (mITT) population, which included all patients who were randomised and received at least one treatment. Sample size calculation is described in the online supplementary file. Enrolment into the $200 \mathrm{mg}$ group was discontinued due to fatalities in patients with SLE who were treated with this dose in a separate trial $^{9}$ (NCT01405196). Power calculations were not impacted for comparisons between PF-04236921 10 or $50 \mathrm{mg}$ and placebo. However, because the $200 \mathrm{mg}$ arm was stopped early, the sample size was less than the planned sample size $(n=40$ instead of 60$)$; hence, the data were not included in the primary efficacy analysis.

Secondary end points in the induction study were CDAI-70 and CDAI-100 response rates, CDAI remission (CDAI score $<150$ ) rates and mean changes from baseline in CDAI scores at weeks 2 through 12. Exploratory end points included comparisons with placebo in the changes from baseline in total scores at week 12 of the Inflammatory Bowel Disease Questionnaire (IBDQ) and the European Quality of Life 5 Dimensions Questionnaire (EQ-5D).

As per protocol, the efficacy, health outcomes and biomarker end points were analysed using the mITT population, defined as all randomised subjects who received at least one dose of PF-04236921. The CDAI response and remission rates at weeks 2 through 12 were analysed using a longitudinal model, described as generalised linear mixed-effects model (statistical methods are discussed in the online supplementary file), containing fixed factors of treatment group, visit and treatment by visit and a random effect for patient and baseline covariates, as per the standard approach for phase II data analyses.

The primary objective of the OLE study was safety. The CDAI instrument that was used to assess efficacy in the induction trial is complicated and intensive. Since efficacy was an exploratory end point in the OLE, the simpler Harvey-Bradshaw Index (HBI; online supplementary table s1) was used to evaluate disease activity and was assessed in all patients who had received at least one dose of PF-04236921 in the OLE (exploratory efficacy end points and analysis are described in the online supplementary file).

The percentage of patients requiring dose escalation, including visit date, was recorded.

Pharmacokinetic, pharmacodynamics and immunogenicity assessments are described in the online supplementary file. 


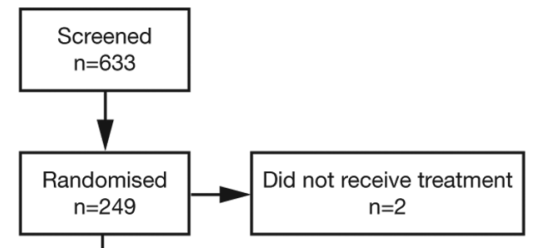

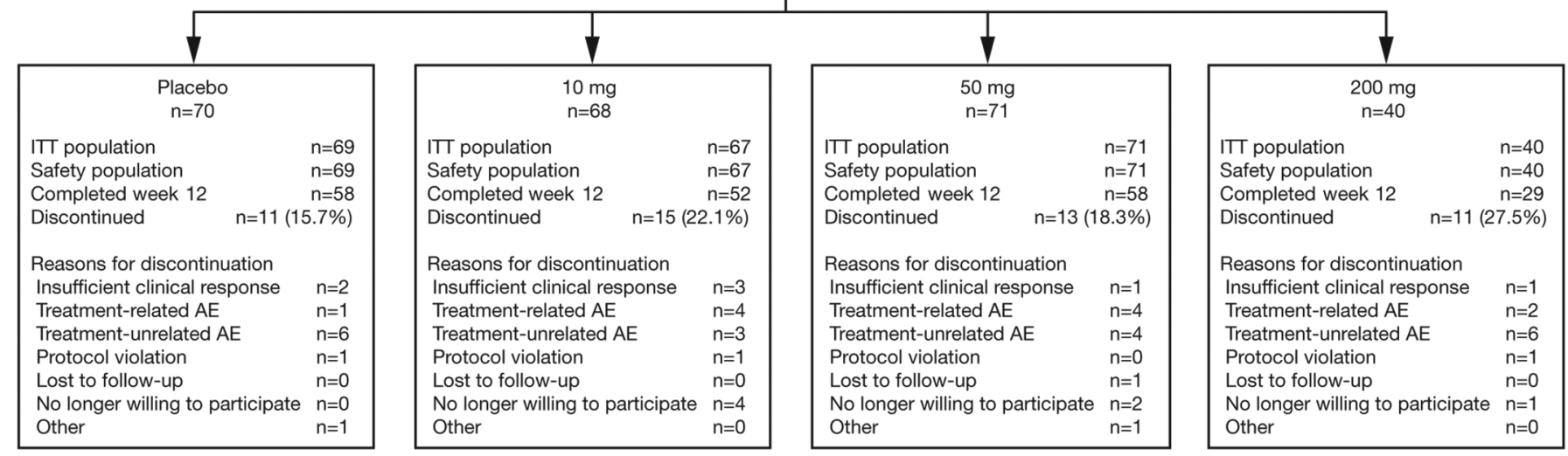

Open-label Extension Study (B0151005)

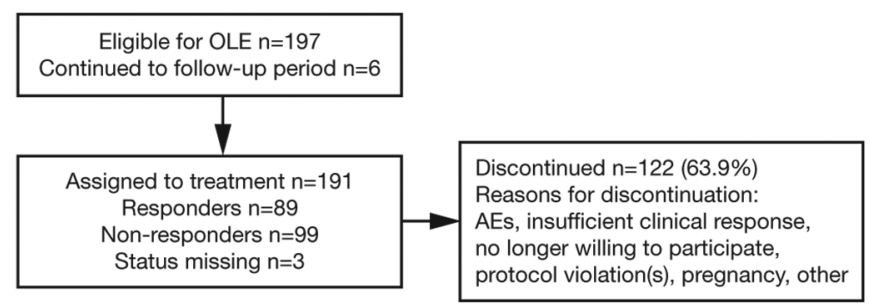

Figure 1 Patient disposition. AE, adverse event; ITT, intent to treat; OLE, open-label extension.

\section{Safety assessments}

Treatment-emergent adverse events (TEAEs) and SAEs, as well as the proportions of patients experiencing events, were reported throughout the study. Other safety considerations are included in the online supplementary file.

\section{RESULTS}

\section{Patient allocation and demographics}

Patients were enrolled from February 2011 to June 2014, with the last patient visit in February 2015 (induction study) and March 2016 (OLE). Of 633 screened patients, 249 were randomised, 247 were included in the safety population and 245 were included in the mITT population (figure 1). Baseline demographics and characteristics were generally balanced between groups (table 1). Patient screen failures, corticosteroid use and endoscopy findings at baseline are described in the online supplementary file.

Fifty patients $(20.1 \%)$ discontinued during the 12 -week treatment period, and the rates were not meaningfully different across the groups, with the exception of the $200 \mathrm{mg}$ group.

In the OLE, 191 patients were enrolled and treated (figure 1), of whom 89 were responders (achieving CDAI-70 at week 12 in the induction study) and 99 non-responders at entry (table 2). Sixty-nine patients $(36.1 \%)$ completed 48 weeks' treatment. The responder and non-responder statuses for completers and discontinued subjects are also provided in table 2 .

\section{Efficacy of induction therapy}

In the induction study, PF-04236921 $50 \mathrm{mg}$ met the primary end point: CDAI-70 response rates were significantly greater than with placebo at weeks $8(49.3 \%$ vs $30.6 \%, \mathrm{P}<0.05)$ and 12 (47.4\% vs $28.6 \%, \mathrm{P}<0.05$ ) (figure 2 ).

Separation from placebo was observed as early as week 4, particularly for CDAI-70 response and remission (figure 3A,C), as well as mean change in CDAI score at weeks 4, 8, 10 and 12 (figure 3D). Notably, CDAI remission rates with PF-04236921 $50 \mathrm{mg}$ were significantly greater than with placebo at week 12 (27.4\% vs $10.9 \%$, respectively) with a difference of $16.5 \%$ $(\mathrm{P}<0.05)$. PF-04236921 $50 \mathrm{mg}$ provided statistically significant improvements over placebo for nearly all time points for CDAI remission (figure 3C,D).

Comparison of $50 \mathrm{mg}$ with placebo for CDAI-100 was significant at week 6 , but not at week 12 (figure $3 \mathrm{~B}$ ).

The $10 \mathrm{mg}$ dose did not meet the primary end point. Numerical improvements were noted in the continuous CDAI score for $10 \mathrm{mg}$ compared with placebo but were not significant (figure 3D). There were no significant differences observed across groups for the number of patients taking oral steroids and/or the median dose usage over time.

A separate longitudinal analysis model was used to evaluate efficacy outcomes for the PF-04236921 $200 \mathrm{mg}$ dose (online supplementary figure s1). Estimates for CDAI-70 response rates at week 8 were $39.0 \%$ with PF-04236921 $200 \mathrm{mg}$ and $28.8 \%$ with placebo; corresponding values at week 12 were $41.7 \%$ and $26.7 \%$, respectively, and were not statistically significant. Results of sensitivity analysis of the efficacy end points and post-hoc subgroup analyses are described in the online supplementary file.

Statistical analyses showed that differences from placebo in the change from baseline in IBDQ total scores ranged from -13.6 to -4.7 for PF-04236921 $10 \mathrm{mg}$ and from -5.7 to 2.2 for $50 \mathrm{mg}$, 
Table 1 Patient demographics and disease characteristics at baseline

\begin{tabular}{|c|c|c|c|c|}
\hline & Placebo $(n=69)$ & $10 \mathrm{mg}(\mathrm{n}=67)$ & $50 \mathrm{mg}(\mathrm{n}=71)$ & $200 \mathrm{mg}(\mathrm{n}=40)$ \\
\hline Mean age, years (SD) & 38.4 (13.6) & 38.9 (12.9) & $38.9(13.1)$ & $42.2(13.2)$ \\
\hline Female, $n(\%)$ & $38(55.1)$ & $34(50.7)$ & $44(62.0)$ & $25(62.5)$ \\
\hline Mean body mass index, $\mathrm{kg} / \mathrm{m}^{2}$ (SD) & $25.8(7.4)$ & $24.8(5.7)$ & $25.0(6.0)$ & $22.9(4.6)$ \\
\hline Median disease duration, years (range) & $10.0(1.2-36.0)$ & $11.0(0.7-49.0)$ & $9.8(0.3-43.0)$ & $11.5(0.3-39.0)$ \\
\hline Mean CDAl score* (SD) & $320.7(64.2)$ & $319.9(61.9)$ & $296.7(63.3)$ & $337.4(73.4)$ \\
\hline Median CRP levels, mg/L (range) & $20.4(0.1-114.7)$ & $16.4(0.1-139.8)$ & $21.1(1.4-106.3)$ & $32.2(1.0-88.4)$ \\
\hline \multicolumn{5}{|l|}{ Prior anti-TNF exposure, $\mathrm{n}(\%)$} \\
\hline Relapsed after at least one anti-TNF & $37(53.6)$ & $33(49.3)$ & $34(47.9)$ & $18(45.0)$ \\
\hline Primary non-responder to at least one anti-TNF & $16(23.2)$ & $15(22.4)$ & $14(19.7)$ & $10(25.0)$ \\
\hline Intolerant to at least one anti-TNF & $11(15.9)$ & $15(22.4)$ & $18(25.4)$ & $9(22.5)$ \\
\hline Discontinued anti-TNF for other reasont & $5(7.2)$ & $4(6.0)$ & $5(7.0)$ & $3(7.5)$ \\
\hline \multicolumn{5}{|l|}{ Current use of immunosuppressive therapy, $\mathrm{n}(\%)$} \\
\hline Azathioprine & $10(14.5)$ & $10(14.9)$ & $13(18.3)$ & $5(12.5)$ \\
\hline 6-Mercaptopurine & $2(2.9)$ & $2(3.0)$ & $3(4.2)$ & $1(2.5)$ \\
\hline Methotrexate & $4(5.8)$ & $8(11.9)$ & $7(9.9)$ & $2(5.0)$ \\
\hline No immunosuppressive therapy & $53(76.8)$ & $47(70.1)$ & $48(67.6)$ & $32(80.0)$ \\
\hline Current use of corticosteroids, $\mathrm{n}$ (\%; median dose) & $35(50.7 ; 20 \mathrm{mg})$ & $17(25.4 ; 15 \mathrm{mg})$ & $29(40.8 ; 20 \mathrm{mg})$ & $19(47.5 ; 20 \mathrm{mg})$ \\
\hline Presence of ulcers, $n(\%)$ & $69(100)$ & $66(98.5)$ & $71(100)$ & $40(100)$ \\
\hline \multicolumn{5}{|l|}{ Sites of involvement } \\
\hline Ileum & $40(58)$ & $48(71.6)$ & $33(46.5)$ & $27(67.5)$ \\
\hline Right colon & $34(49.3)$ & $25(37.3)$ & $28(39.4)$ & $19(47.5)$ \\
\hline Transverse colon & $30(43.5)$ & $23(34.3)$ & $34(47.9)$ & $21(52.5)$ \\
\hline Left colon & $46(66.7)$ & $32(47.8)$ & $50(70.4)$ & $29(72.5)$ \\
\hline Rectum & $38(55.1)$ & $30(44.8)$ & $47(66.2)$ & $23(57.5)$ \\
\hline \multicolumn{5}{|l|}{ SES-CD subscores, mean (SD) } \\
\hline Ileum & $3.9(3.5)(n=67)$ & $4.4(3.4)(n=66)$ & $3.6(3.8)(n=68)$ & $4.6(3.7)(n=40)$ \\
\hline Right colon & $3.0(3.4)(n=66)$ & $2.2(2.7)(n=66)$ & $2.2(2.8)(n=67)$ & $2.8(3.2)(n=39)$ \\
\hline Transverse colon & $2.5(3.1)(n=68)$ & $2.0(2.9)(n=67)$ & $2.8(3.3)(n=70)$ & $3.4(3.4)(n=39)$ \\
\hline Left colon & $4.0(3.4)(n=69)$ & $3.2(3.6)(n=67)$ & $4.6(3.3)(n=70)$ & $5.1(3.8)(n=40)$ \\
\hline Rectum & $3.4(3.4)(n=69)$ & $3.1(3.5)(n=67)$ & $4.1(3.4)(n=71)$ & $3.8(3.7)(n=40)$ \\
\hline
\end{tabular}

${ }^{*}$ The mean baseline CDAl score was different between the $50 \mathrm{mg}$ and placebo groups, and this difference was statistically significant.

†This category captures discontinuations unrelated to efficacy or safety and may include financial/insurance-based issues to continuing the treatment.

CDAI, Crohn's Disease Activity Index; CRP, C-reactive protein; SES-CD, Simple Endoscopic Score for CD; TNF, tumour necrosis factor.

\begin{tabular}{|ll}
\hline Table 2 & Patient disposition in the open-label extension study \\
\hline & PF-04236921 \\
\hline Enrolled and treated* $^{*}$ & 191 \\
\hline Responder at entry & 89 \\
\hline Non-responder at entry & 99 \\
\hline Responder status missing at entry & 3 \\
\hline End of treatment: completed & 69 \\
\hline Baseline HBI data & 65 \\
\hline Responders & 36 \\
\hline Non-responders & 29 \\
\hline End of treatment: discontinued & 122 \\
\hline Baseline HBI data & 121 \\
\hline Responders & 51 \\
\hline Non-responders & 70 \\
\hline End of study (includes follow-up period): completed & 111 \\
\hline Analysed for safety & 191 \\
\hline Analysed for efficacy & $189 \dagger$ \\
\hline
\end{tabular}

*Patients in the prior induction study achieving CDAI-70 (proportion of patients who achieved a $\geq 70$-point reduction in Crohn's Disease Activity Index (CDAI) score) response were considered responders at entry and those patients not achieving CDAI-70 response were considered non-responders at entry.

tPatients who received at least one dose of PF-04236921 in this study but excludes two patients with a quality issue.

HBI, Harvey-Bradshaw Index. and were not statistically significant (one-sided P values $\geq 0.05$ ) at weeks 4,8 and 12 . At the same time points, differences from placebo in the change from baseline in the EQ-5D utility index ranged from -0.013 to 0.018 for PF-04236921 $10 \mathrm{mg}$ and from 0.011 to 0.043 for $50 \mathrm{mg}$, and were not statistically significant (one-sided $\mathrm{P}$ values $\geq 0.05$ ).

\section{Long-term effects of PF-04236921}

For the 191 patients who entered the OLE study, 89 (46\%) were assessed as CDAI responders at OLE baseline, and 31\% were CDAI remitters at baseline. As an exploratory endpoint, maintenance of response by PF-04236921 was assessed using HBI. For the subset of patients who had been on active therapy and were responders at baseline for the OLE $(n=65)$, the response and remission rates were $40 \%$ (online supplementary figure s3) and $32 \%$, respectively, by week 48 of the OLE.

For the overall OLE population $(\mathrm{n}=191), 77.8 \%$ of patients (SE 0.073) had their dose escalated to $100 \mathrm{mg}$ between weeks 8 and 48. Time to dose escalation is shown in online supplementary figure $\mathrm{s} 4$.

\section{Pharmacokinetic and pharmacodynamic outcomes}

Changes in serum concentrations of PF-04236921 and CRP in the induction trial (online supplementary figures $\mathrm{s} 5$ and s6) and changes in serum concentrations of PF-04236921 in the OLE 


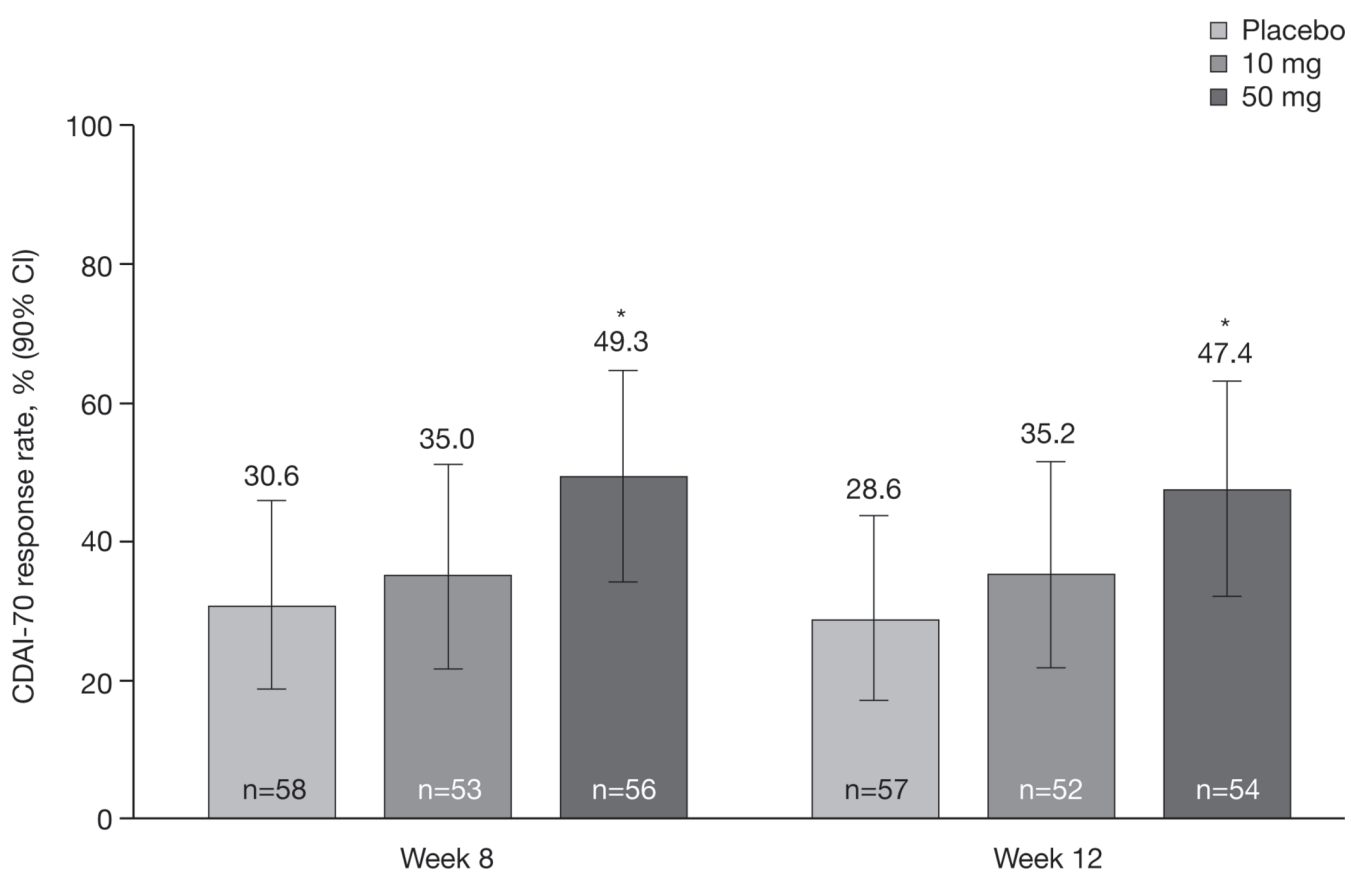

Figure 2 Primary end point: CDAI-70 response rates at weeks 8 and 12 (generalised linear mixed model; modified intention-to-treat population). ${ }^{*} \mathrm{P}<0.05$ versus placebo. CDAI, Crohn's Disease Activity Index; CDAI-70, proportion of patients who achieved a $\geq 70$-point reduction in CDAI score.

(online supplementary figure s7) are described in the online supplementary file.

Identification of antidrug antibodies (ADAs) was rare across the two studies. Of 680 serum samples collected for ADA analysis in the induction study, one $(0.1 \%)$ was confirmed as ADA-positive $(50 \mathrm{mg}$, week 4$)$ and also tested positive for neutralising antibodies (Nab). These findings did not appear to be associated with any TEAEs or pharmacokinetic effects in this patient. In the OLE study, among 1159 serum samples, six samples from one patient were confirmed ADA positive and 1/6 was confirmed Nab-positive. There was no discernible impact of this positive ADA result on the patient's pharmacokinetic profile.

Serum CRP levels were continuously suppressed during the treatment period in the OLE study, returning to baseline during the follow-up period. At the OLE baseline, the median serum concentration of CRP was $4.030 \mathrm{mg} / \mathrm{mL}(-74.3 \%$ change from induction study baseline). The median per cent changes from induction study baseline in serum concentrations of CRP were $-93.5 \%$ and $-89.2 \%$, respectively, at weeks 4 and 48 . By the end of the study (week 76), the median per cent change from induction study baseline was $-57.9 \%$.

Results from faecal calprotectin assays were highly variable at baseline and over time in all groups. No significant treatment effects of PF-04236921 on faecal calprotectin were identified.

\section{Safety outcomes}

Rates of TEAE and SAEs are described in table 3 and online supplementary table s2 for the induction and OLE studies, respectively. For both studies, the most frequent TEAEs and SAEs with PF-04236921 were CD related (including worsening, exacerbation and flare of $\mathrm{CD}$ and abdominal pain) and nasopharyngitis.

During the OLE study, there were 890 AEs in 171 patients $(89.5 \%)$ and most AEs were mild or moderate in severity. A total of 58 patients $(30.4 \%)$ experienced SAEs of all causalities in the treatment period of the OLE study.
There was one death in the induction study, which occurred in the $50 \mathrm{mg}$ group, in a patient in their mid-70s who had a 54-year history of ileocolonic CD without prior surgery and a medical history of chronic obstructive pulmonary disease. The patient died of respiratory failure secondary to pneumonia on day 96 , judged as unrelated to treatment by the investigator. The patient had a colectomy on day 80 and experienced a postoperative complication of respiratory failure. The full narrative can be found in the online supplementary file. There were no deaths in the OLE.

Injection-site TEAEs were infrequent (placebo, $\mathrm{n}=4$; PF-04236921 $10 \mathrm{mg}, \mathrm{n}=2 ; 50 \mathrm{mg}, \mathrm{n}=7 ; 200 \mathrm{mg}, \mathrm{n}=6$ ) in the induction study, and there were 42 in the OLE.

In light of the concern for risk of GI perforation and abscess with this mechanism of action (and because these events may be reported by investigators as infections or GI events), online supplementary table 33 lists the events of abscess and perforation identified by the Standardised Medical Dictionary for Regulatory Activities Queries search. During the induction study, six patients experienced seven SAEs of abscess or perforation, all in PF-04236921-treated patients. In the OLE study treatment period, 10 patients experienced 12 SAEs of abscess and perforation (50 mg, $\mathrm{n}=6 ; 100 \mathrm{mg}, \mathrm{n}=4)$.

\section{DISCUSSION}

The phase II induction trial supports the efficacy of PF-04236921 $50 \mathrm{mg}$ SC in anti-TNF-experienced patients with moderate-to-severe $\mathrm{CD}$, administered on days 1 and 28, for inducing response and remission over 12 weeks. The $50 \mathrm{mg}$ dose met the primary end point, significantly improving CDAI-70 response rates versus placebo at weeks 8 and 12. At the time the study was designed, the use of the CDAI instrument for the primary end point was standard. Using the more rigorous CDAI end point(s), CDAI remission for the $50 \mathrm{mg}$ dose was significant when compared with placebo at weeks 8 and 12, but the CDAI-100 did not reach significance at these time points. While randomisation 
A

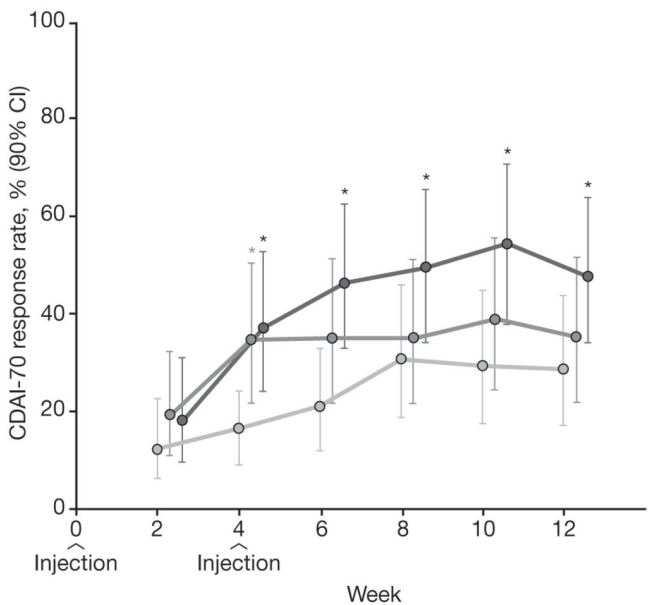

B

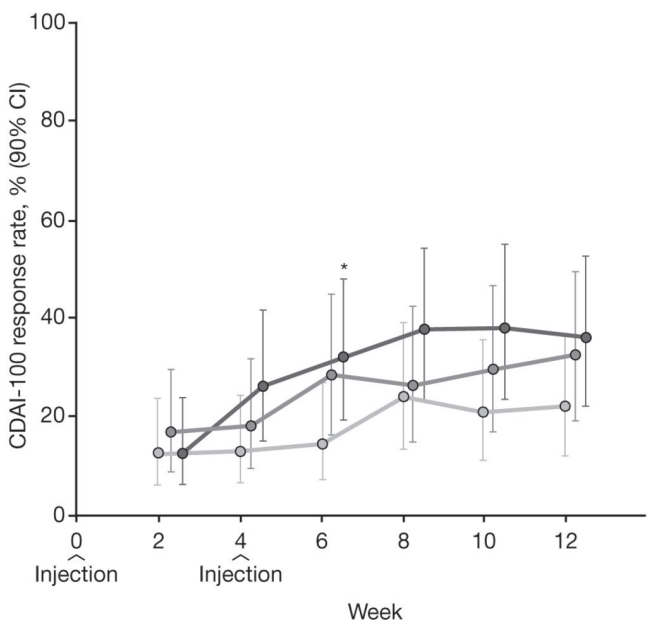

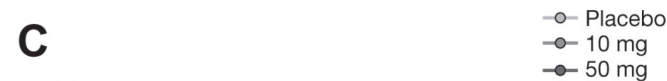

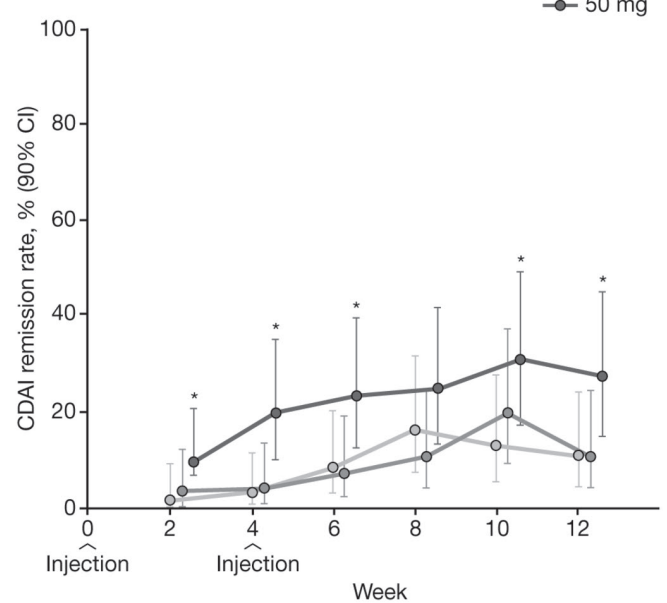

D

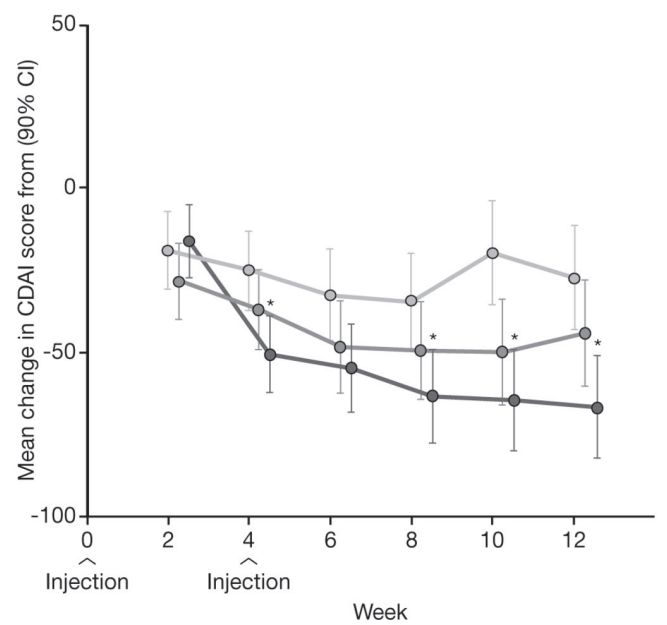

Figure 3 CDAl outcomes from week 2 to week 12 by treatment group: (A) CDAI-70 response rate; (B) CDAI-100 response rate; (C) CDAI remission rate; and (D) mean changes in CDAI score from baseline (generalised linear mixed model; modified intention-to-treat population). ${ }^{*} P<0.05$ versus placebo. CDAI, Crohn's Disease Activity Index; CDAI-70/100, proportion of patients who achieved a 70/100-point reduction in CDAI score.

was generally balanced across the arms, there was a numerically lower baseline CDAI score in the $50 \mathrm{mg}$ group that may have contributed to the lower CDAI-100 response rates in that group (eg, subjects who entered the study with scores between 220 and 250 would be unable to numerically achieve a 100-point decrease). The changes in continuous CDAI score are supportive of the rapid onset of action with separation from placebo as early as week 4 and maintained through week 12 . Newer end points for CD that include measures of mucosal healing cannot be discerned from this study as endoscopy was not performed in a systematic manner.

The primary analysis method for this trial was a longitudinal method that incorporated all data, even from those patients who discontinued prior to week 12 . The non-responder imputation (NRI) analysis is cross-sectional (using responder data only from week 12) that is more commonly used in phase III confirmatory studies, where the sample size is substantially larger. In addition, the NRI imputes any missing data as non-responder, which is the most conservative form of imputation. The longitudinal mixed-effect model repeat measurement method is considered to be more powerful than the NRI method for this exploratory phase II study. Nonetheless, as a post-hoc analysis, the data were reanalysed using the NRI approach. The difference between $50 \mathrm{mg}$ and placebo at week 12 for CDAI-70 was $8 \%$ (95\% CI $-6 \%$ to $21 \%$ ) and for CDAI remission was $14 \%(95 \%$ CI 0.03 to 0.24 ) (online supplementary table s4).

In this CD study, the early termination of the $200 \mathrm{mg}$ dose group resulted in a much smaller sample size. Given this limitation, there did not appear to be additional benefit for the $200 \mathrm{mg}$ dose to balance the higher rates of SAEs and discontinuations due to TEAEs.

For patients who entered the OLE trial as responders and who had been on active drug in the induction period $(n=65)$, the HBI response and remission rates were $40 \%$ and $32 \%$ at week 48 . By inspection of PF-04236921 concentrations, the loss of response over time in more than half of the patients did not appear to be associated with decrease of drug levels and/or with occurrence of ADAs, as has been commonly observed for other anticytokine therapies. Maintenance for patients induced on anti-IL-6 therapy is impacted by attenuation of unknown cause and will require development of new optimisation of treatment strategies that could include high(er) dose and/or longer duration of induction or alternate drug combinations in order to avoid immunological breakthroughs.

Occurrence of GI perforation has been reported in patients with rheumatoid arthritis treated with a humanised monoclonal antibody against the IL-6 receptor, tocilizumab. ${ }^{10} 11$ Most cases 
Table 3 Treatment-emergent adverse events during the 12-week induction trial

\begin{tabular}{|c|c|c|c|c|}
\hline & Placebo $(n=69)$ & $10 \mathrm{mg}(\mathrm{n}=67)$ & $50 \mathrm{mg}(\mathrm{n}=71)$ & $200 \mathrm{mg}(\mathrm{n}=40)$ \\
\hline Any adverse events, $n(\%)$ & $63(91.3)$ & $60(89.6)$ & $58(81.7)$ & $33(82.5)$ \\
\hline Severe adverse events, n (\%) & $5(7.2)$ & $12(17.9)$ & $12(16.9)$ & $5(12.5)$ \\
\hline Serious adverse events, $\mathrm{n}(\%)$ & $9(13.0)$ & $7(10.4)$ & $9(12.7)$ & $11(27.5)$ \\
\hline GI disorders* & $5(7.2)$ & $3(4.5)$ & $7(9.9)$ & $8(20.0)$ \\
\hline \multicolumn{5}{|l|}{ Serious infections and infestations } \\
\hline Sepsis & $1(1.4)$ & - & - & - \\
\hline Anal abscess & - & $1(1.5)$ & $2(2.8)$ & - \\
\hline Groin abscess & - & - & - & $1(2.5)$ \\
\hline Intestinal abscess & - & $1(1.5)$ & - & - \\
\hline Pneumonia & - & - & - & $1(2.5)$ \\
\hline Deaths & $0(0.0)$ & $0(0.0)$ & $1(1.4)$ & $0(0.0)$ \\
\hline Discontinuations due to adverse events, $\mathrm{n}(\%)$ & $7(10.1)$ & $6(9.0)$ & $6(8.5)$ & $8(20.0)$ \\
\hline Temporary discontinuation due to adverse events, $\mathrm{n}(\%)$ & $0(0.0)$ & $0(0.0)$ & $1(1.4)$ & $0(0.0)$ \\
\hline \multicolumn{5}{|l|}{ Common adverse events ( $\geq 5 \%$ in any treatment group), $n(\%)$} \\
\hline Crohn's diseaset & $8(11.6)$ & $3(4.5)$ & $10(14.1)$ & $8(20.0)$ \\
\hline Abdominal pain & $8(11.6)$ & $6(9.0)$ & $8(11.3)$ & $6(15.0)$ \\
\hline Headache & $6(8.7)$ & $5(7.5)$ & $8(11.3)$ & $2(5.0)$ \\
\hline Nasopharyngitis & $3(4.3)$ & $10(14.9)$ & $7(9.9)$ & $3(7.5)$ \\
\hline Nausea & $1(1.4)$ & $7(10.4)$ & $7(9.9)$ & $1(2.5)$ \\
\hline Rash & $1(1.4)$ & $2(3.0)$ & $7(9.9)$ & $1(2.5)$ \\
\hline Arthralgia & $8(11.6)$ & $5(7.5)$ & $5(7.0)$ & $0(0.0)$ \\
\hline Erythema & $0(0.0)$ & $1(1.5)$ & $4(5.6)$ & $1(2.5)$ \\
\hline Fatigue & $0(0.0)$ & $2(3.0)$ & $4(5.6)$ & $0(0.0)$ \\
\hline Proctalgia & $0(0.0)$ & $2(3.0)$ & $4(5.6)$ & $0(0.0)$ \\
\hline Pyrexia & $8(11.6)$ & $5(7.5)$ & $4(5.6)$ & $1(2.5)$ \\
\hline Upper respiratory tract infection & $2(2.9)$ & $0(0.0)$ & $4(5.6)$ & $0(0.0)$ \\
\hline Back pain & $4(5.8)$ & $4(6.0)$ & $3(4.2)$ & $1(2.5)$ \\
\hline Peripheral oedema & $2(2.9)$ & $2(3.0)$ & $3(4.2)$ & $2(5.0)$ \\
\hline Urinary tract infection & $3(4.3)$ & $2(3.0)$ & $3(4.2)$ & $5(12.5)$ \\
\hline Vomiting & $2(2.9)$ & $3(4.5)$ & $3(4.2)$ & $2(5.0)$ \\
\hline Gastroenteritis & $1(1.4)$ & $0(0.0)$ & $1(1.4)$ & $2(5.0)$ \\
\hline Constipation & $2(2.9)$ & $2(3.0)$ & $0(0.0)$ & $2(5.0)$ \\
\hline Upper abdominal pain & $0(0.0)$ & $1(1.5)$ & $0(0.0)$ & $3(7.5)$ \\
\hline
\end{tabular}

${ }^{*} \mathrm{Gl}$ disorders include Crohn's disease, abdominal pain, anal fistula, intestinal perforation, intestinal stenosis, colitis, hematochezia and acute pancreatitis.

tIncludes worsening, exacerbation and flare of Crohn's disease.

occurred in patients with diverticulitis and those receiving non-steroidal anti-inflammatory drugs and/or long-term corticosteroids. ${ }^{11}$ Given this observation, as well as the association between CD and GI perforation, fistula formation and abscess, ${ }^{12}$ GI perforation was a potential safety concern for PF-04236921 in CD. To reduce the risk, patients with a history of diverticulitis and patients with active fistulae or abscess were excluded. Patients were allowed to enrol in this study if computed tomography-MRI was performed within 6 months of screening to exclude active fistula or abscess. We acknowledge that imaging conducted during the screening period may have been more appropriate to exclude patients with abscess or fistula particularly because many of the participants in this trial had extensive disease history (median disease duration approximately 10 years). There were cases of GI perforation and GI abscesses observed in both the induction and OLE studies. In the induction period, there did not appear to be an increase in frequency of these events with dose, although all six patients with events were in the PF-04236921-treated groups in the induction period. The interpretation of dose dependency in the OLE is complicated by: the lack of a placebo control; subjects having entered the study from different dose levels (including placebo); and the fact that a one-time dose escalation was allowed in the OLE.
The DMC reviewed all cases and did not recommend changes in treatment. Most cases of GI perforation or abscess were identified in regions of previous disease involvement and/or surgery, making it difficult to discriminate the impact of disease progression versus study drug. Nonetheless, because the events occurred only in the PF-04236921 groups (compared with placebo), this represents a signal that requires careful attention in future clinical trials.

Although anti-TNF therapy represents a significant landmark in the treatment of $\mathrm{CD}$, many patients experience primary non-response or relapse. ${ }^{5}$ The present study suggests that targeting IL-6 may provide a further therapeutic option for this population in whom CDAI response and remission rates for a second biologic are modest, as shown in trials with vedolizumab $(44 \%$ and $39 \%$, respectively, in a population of $50 \%$ anti-TNF-experienced patients), ${ }^{13}$ ustekinumab (59\% and $53 \%$, respectively, in a broad population; and $41 \%$ remission in the anti-TNF-experienced subgroup $)^{14}$ and adalimumab (43\% [CDAI-70] and 36\%, respectively, in a broad population) ${ }^{1}$ at the approximate 1-year end point (values are not placebo adjusted to allow comparison with results in this study). The outcomes with PF-04236921 are particularly encouraging in the context of a very difficult-to-treat population: in addition to inadequate 
response to one or more prior anti-TNF therapies, patients had a median disease duration of approximately 10 years and many had undergone previous resection. Patients with high baseline CRP levels $(\geq 5.0 \mathrm{mg} / \mathrm{L})$ were enrolled (elevated CRP transcriptionally controlled by IL-6) because this population may be expected to be more susceptible to anti-IL- 6 therapy. ${ }^{6} 8$

In the induction study, the reduction in serum CRP (a pharmacodynamic marker of IL-6 suppression) appeared to increase monotonically as dose increased, reaching 95\% reduction from baseline for the $200 \mathrm{mg}$ dose group at week 12 . The $50 \mathrm{mg}$ dose was associated with a median reduction of $86.3 \%$ from baseline and was the most effective dose in the induction study based on CDAI. From the available data, it is not clear that the additional pharmacological suppression provided by $200 \mathrm{mg}$ leads to greater benefit compared with $50 \mathrm{mg}$. No significant changes were observed in the faecal calprotectin marker for any of the dose groups.

The $50 \mathrm{mg}$ dose group met the primary end point for CDAI-70 response rate in this study and demonstrated statistical significance for the secondary end point of CDAI remission rate. The overall benefit/risk profile for PF-04236921 appears to be acceptable for the continued development of this treatment in this refractory CD population. However, the signals of GI perforation and abscess do require careful consideration and characterisation during future clinical development.

\author{
Author affiliations \\ ${ }^{1}$ Gastrointestinal Immunopathology Lab and IBD Unit, Humanitas Clinical and \\ Research Center and Humanitas University, Milan, Italy \\ ${ }^{2}$ Department of Gastroenterology, University Hospitals Leuven, Leuven, Belgium \\ ${ }^{3}$ Nature Coast Clinical Research, Inverness, Florida, USA \\ ${ }^{4}$ Inflammatory Bowel Disease Clinic, University of Calgary, Calgary, Canada \\ ${ }^{5}$ Department of Gastroenterology and Hepatology, University of Zürich, Zürich, \\ Switzerland \\ ${ }^{6}$ Division of Gastroenterology, Rabin Medical Center, University of Tel-Aviv, Petah \\ Tikva, Israel \\ ${ }^{7}$ UOC Gastroenterologia, AO San Camillo Forlanini, Rome, Italy \\ ${ }^{8}$ Lille University School of Medicine, University of Lille, Inserm U995, Lille, France \\ IInflammatory Bowel Diseases Service, Concord Hospital, Sydney, New South Wales, \\ Australia \\ ${ }^{10}$ Formerly of Worldwide Research and Development, Pfizer Inc, Cambridge, \\ Massachusetts, USA \\ ${ }^{11}$ Worldwide Research and Development, Pfizer Inc, Cambridge, Massachusetts, USA \\ ${ }^{12}$ Worldwide Research and Development, Pfizer Inc, Collegeville, Pennsylvania, USA \\ ${ }^{13}$ Department of Medicine I, Christian-Albrechts-University and University Hospital \\ Schleswig-Holstein, Kiel, Germany
}

Acknowledgements The authors would like to thank the patients who were involved in these studies, the study investigators and the study team.

Contributors SD contributed to study concept and design, acquisition of data, interpretation of data, drafting of the manuscript, critical review of the manuscript, statistical analysis, other support (administrative, technical, material) and study supervision. SV, GR and AK contributed to critical review of the manuscript. PH conducted the study per protocol, contributed to patient acquisition and drafting of the manuscript. RP, GF and NR contributed to interpretation of data, drafting of the manuscript and critical review of the manuscript. PD contributed to acquisition of data, interpretation of data, drafting of the manuscript and critical review of the manuscript. RWL contributed to drafting of the manuscript and critical review of the manuscript. GMC, MKM and JB contributed to study concept and design, interpretation of data, drafting of the manuscript and critical review of the manuscript. FC performed data analyses and contributed to study concept and design, acquisition of data, interpretation of data, drafting of the manuscript, critical review of the manuscript, statistical analysis and other support (administrative, technical, material) and led study supervision. AB performed statistical analysis, interpretation of data, drafting of the manuscript and critical review of the manuscript. $\mathrm{CL}$ contributed to study concept and design and performed data analyses. SS contributed to study concept and design, acquisition of data, interpretation of data, drafting of the manuscript and critical review of the manuscript.

Funding Both studies were sponsored by Pfizer. Authors employed by Pfizer Inc were involved in the study design and in the collection, analysis and interpretation of data. Editorial support under the direction of the authors was provided by Hannah FitzGibbon, PhD, and Neil Cockburn, BSc, of Complete Medical Communications, and funded by Pfizer.

Disclaimer GC and FC were employees of Worldwide Research and Development, Pfizer Inc, Cambridge, MA, USA, at the time of the study.

Competing interests SD is a speaker, consultant and advisory board member for Abbott Laboratories, AbbVie, Actelion, Alphawasserman, AstraZeneca, Cellerix, Cosmo Pharmaceuticals, Ferring, Genentech, Grunenthal, Hospira, Johnson and Johnson, Merck \& Co, Millennium Takeda, Mundipharma, Novo Nordisk, Nycomed, Pharmacosmos, Pfizer, Schering-Plough, UCB Pharma and Vifor. SV received research support from AbbVie, MSD and Takeda and received consultancy and/or speaker honoraria from AbbVie, Celgene, Centocor, Ferring, Galapagos, Genentech/Roche, Hospira, MSD, Mundipharma, Pfizer, Shire and Takeda. PH is a consultant and/ or advisory board participant for, and/or has received speaker honoraria, research support and/or educational grants from, Cubist, Evoke, Ferring, Forest, Furiex, Gilead, GlaxoSmithKline, Intercept, Ironwood, Menarini, Nature Coast Clinical Research, Pfizer, Rhythm, Salix, Synergy, Takeda, Theravance and Vivus. RP is a consultant for and/or received lecture fees from Abbott, AbbVie, Allergan, Amgen, AstraZeneca, Axcan Pharma, Biogen Idec, Bristol-Myers Squibb, Centocor, ChemoCentryx, Eisai Medical Research, ELAN, Ferring, Genentech, GlaxoSmithKline, Janssen, Millennium, MSD, Ocera Therapeutics, Otsuka America Pharmaceuticals, Pfizer, Shire Pharmaceuticals, Prometheus, Robarts Clinical Trials, Schering-Plough, Synta Pharmaceuticals, Takeda, Teva, UCB Pharma and Warner Chilcott. GR is a consultant for Abbott, AbbVie, Augurix, Boehringer, Calypso, FALK, Ferring, Fisher, Genentech, Essex/MSD, Novartis, Pfizer, Phadia, Roche, Takeda, Tillots, UCB, Vifor, Vital Solutions and Zeller; received speaker honoraria from AstraZeneca, Abbott, AbbVie, FALK, MSD, Phadia, Tillots, UCB and Vifor; and received educational grants and research grants from Abbot, AbbVie, Ardeypharm, Augurix, Calypso, Essex/MSD, FALK, Flamentera, Novartis, Roche, Takeda, Tillots, UCB and Zeller. AK received educational grants from AbbVie, MSD and Takeda. PD participated in advisory boards for, and received fees and grants from, AbbVie, Biofortis, BioMérieux Danone, Ferring, Genfit, Intralytix, Kitozyme, Lesaffre, MSD, Norgine, OmegaPharma International, Pileje, PPM Roquette, Takeda and TxCell. RWL participated in advisory boards for AbbVie, Aspen, Ferring, Janssen, Hospira and Takeda and received research support from Janssen, NHMRC Career Development Fellowship and Shire. SS received consultancy fees from AbbVie, Bristol-Myers Squibb, Boehringer, Ferring, Genentech/Roche, Pfizer, MSD/Janssen, Takeda and UCB and lecture fees from AbbVie, MSD/Janssen, Takeda and UCB. AB, MKM, CL, NR and JB are all employees of Pfizer Inc and hold stock or options in Pfizer Inc. GMC and FC are former employees of Pfizer Inc and FC is a current employee of Shire Pharmaceuticals. GF has no conflicts of interest to report. GMC has been a consultant for Adare, Albireo, AstraZeneca, CinRx, Cutis, OrphoMed, Second Genome, Strongbridge and Zealand.

Ethics approval Institutional review board.

Provenance and peer review Not commissioned; externally peer reviewed.

Author note $\mathrm{GC}$ and FC were employees of Worldwide Research and Development, Pfizer Inc, Cambridge, MA, USA, at the time of the study.

Open access This is an open access article distributed in accordance with the Creative Commons Attribution Non Commercial (CC BY-NC 4.0) license, which permits others to distribute, remix, adapt, build upon this work non-commercially, and license their derivative works on different terms, provided the original work is properly cited and the use is non-commercial. See: http://creativecommons.org/ licenses/by-nc/4.0/

(c) Article author(s) (or their employer(s) unless otherwise stated in the text of the article) 2019. All rights reserved. No commercial use is permitted unless otherwise expressly granted.

\section{REFERENCES}

1 Colombel JF, Sandborn WJ, Rutgeerts P, et al. Adalimumab for maintenance of clinical response and remission in patients with Crohn's disease: the CHARM trial. Gastroenterology 2007;132:52-65.

2 Hanauer SB, Feagan BG, Lichtenstein GR, et al. Maintenance infliximab for Crohn's disease: the ACCENT I randomised trial. Lancet 2002;359:1541-9.

3 Sandborn WJ, Feagan BG, Stoinov S, et al. Certolizumab pegol for the treatment of Crohn's disease. N Engl J Med 2007;357:228-38.

4 Targan SR, Feagan BG, Fedorak RN, et al. Natalizumab for the treatment of active Crohn's disease: results of the ENCORE Trial. Gastroenterology 2007;132:1672-83.

5 Ding NS, Hart A, De Cruz P. Systematic review: predicting and optimising response to anti-TNF therapy in Crohn's disease - algorithm for practical management. Aliment Pharmacol Ther 2016;43:30-51.

6 Allocca M, Jovani M, Fiorino G, et al. Anti-IL-6 treatment for inflammatory bowel diseases: next cytokine, next target. Curr Drug Targets 2013;14:1508-21.

7 Ito H, Takazoe M, Fukuda Y, et al. A pilot randomized trial of a human anti-interleukin-6 receptor monoclonal antibody in active Crohn's disease. Gastroenterology 2004;126:989-96. 
8 Fogel R, Sridharan S, Li C, et al. AB0724 Safety, pharmacokinetics, and pharmacodynamics of a human anti-IL6 monoclonal antibody PF-04236921 in healthy subjects. Ann Rheum Dis 2013;71 (Suppl.3):680.

9 Wallace DJ, Strand V, Merrill JT, et al. Efficacy and safety of an interleukin 6 monoclonal antibody for the treatment of systemic lupus erythematosus: a phase II dose-ranging randomised controlled trial. Ann Rheum Dis 2017;76:534-42.

10 Curtis JR, Perez-Gutthann S, Suissa S, et al. Tocilizumab in rheumatoid arthritis: a case study of safety evaluations of a large postmarketing data set from multiple data sources. Semin Arthritis Rheum 2015;44:381-8.
11 Gout T, Ostör AJ, Nisar MK. Lower gastrointestinal perforation in rheumatoid arthritis patients treated with conventional DMARDs or tocilizumab: a systematic literature review. Clin Rheumatol 2011;30:1471-4.

12 Wilkins T, Jarvis K, Patel J. Diagnosis and management of Crohn's disease. Am Fam Physician 2011;84:1365-75.

13 Sandborn WJ, Feagan BG, Rutgeerts P, et al. Vedolizumab as induction and maintenance therapy for Crohn's disease. N Engl J Med 2013;369:711-21.

14 Feagan BG, Sandborn WJ, Gasink C, et al. Ustekinumab as Induction and Maintenance Therapy for Crohn's Disease. N Engl J Med 2016;375:1946-60. 